\title{
Letter to the Editor in Response to: Achieving a High Level of Protection from Pesticides in Europe: Problems with the Current Risk Assessment Procedure and Solutions
}

\author{
Anthony C. TWEEDALE* ${ }^{*}$
}

\section{Dear Editor:}

Robinson et al (2020) ${ }^{1}$ make a set of crucially misdirected, irrelevant criticisms of what they and I agree is the failure to implement the world's most progressive chemicals regulation, the European Union's (EU) pesticide market authorisation regulation, EC 1107/2009. While they begin with the real problem, they severely underestimate it: uniquely in the EU, both the general chemicals regulation REACh and the pesticides regulation require that industry and regulators evaluate, in hazard or risk assessments (RA), "all available information" (AAI) (or at least AAI since the previous authorisation of a pesticide). This crucially includes the published toxicity findings of academia. RA is a classic GIGO (garbage in = garbage out) linear process: hazard/risk can only be known if all data are evaluated: "Literature searches underlie the foundations of systematic reviews and related review types."2 Or as the US National Academies of Science put it in their seminal report creating the world's method for performing RA, the 1983 'Red Book': "Risk assessment is the use of the factual base to define the health effects of exposure of individuals or populations to hazardous materials and situations". 3

\footnotetext{
Rebutting Industry Science with Knowledge (RISK) Consultancy, Brussels, Belgium; email: ttweed@telenet.be.

1 C Robinson, CJ Portier, A \avoški, R Mesnage, A Roger, P Clausing, P Whaley, H Muilerman and A Lyssimachou, "Achieving a high level of protection from pesticides in Europe: problems with the current risk assessment procedure and solutions" (2020) 11(3) European Journal of Risk Regulation 450-80.

2 ML Rethlefsen, S Kirtley, S Waffenschmidt et al, "PRISMA-S: an extension to the PRISMA Statement for Reporting Literature Searches in Systematic Reviews” (2021) 10 Systematic Reviews 39 (emphasis added).

3 National Research Council (US), Risk Assessment in the Federal Government: Managing the Process (Washington, DC: National Academies Press 1983) (emphasis added).
} 
Far beyond Robinson et al's single citation, three more audits ${ }^{4}$ prove this all-important fact: industry and regulators are ignoring the AAI mandate. Extrapolating from those random samples, dozens of pesticides and hundreds of high-volume REACh chemicals are authorised to market without having evaluated a single one of their published toxicity findings by academics (where each has from dozens to thousands); they average finding under $20 \%$ of the most easily found ones. All of those that are found get zero to minimal evaluations, with rare exception when a controversy erupts. Never in a RA done to allow a chemical to enter or remain on the market has the toxicity finding of an academic been the key study (ie to set the "safe dose"); a conference of 250 global risk assessors could not name one to me.

The technical RA problems that Robinson et al devote the most words to - industry's efforts to minimise test requirements - are: (1) downstream of AAI; (2) have nothing to do with the core of RA; and (3) would be improved by fixing the upstream AAI problem. If these were addressed, academics and their allies would finally be able to show and debate with regulators regarding their contrasting methods and findings. I estimate that $1,000+$ low-dose in vertebrate findings appear yearly, and this is accelerating because biochemistry evolved low signal strengths in the crowded environment in and around cells, so that low doses of disrupting synthetic chemicals can have big effects.

But without such findings at hand, regulators and politicians will continue to say: “... in order to fulfil our legal health mandates, we must rely on the most accurate data available" (paraphrase of a recent EU health commissioner). What he was referring to was industry's utterly insensitive toxicity test methods. For example, I was personally informed by the Commission (COM) that the reason COM exempted industry's tests from having to undergo systematic review (the gold standard of RA) in its new regulation to determine which chemicals are endocrine disruptors is that "they have already been deemed to be reliable"; in other words, the Organisation for Economic Co-operation and Development's (OECD) standardised "test guideline" (TG) test methods are de facto reliable (every stakeholder ignored this example of the AAI problem). "Reliable" obviously means "accurate", and accuracy requires both sensitivity and specificity (true-positive and true-negative results). For health, false-positive results are a bearable price for being sensitive enough to detect toxicity.

The overwhelming insensitivity of industry/regulator's TGs (for they were donated to OECD by industry) is that they literally do not even test their hypothesis of "what are the effects of our exposure?" because their chronic exposure doses are unrealistically high, being derived from (and much closer to) acute (poisonous) doses. When academics test that hypothesis, tens of thousands of low-dose toxicity findings result (and their rate is

4 The one cited near the start of Robinson et al, and:

(1) Generations Futures, "Carences des évaluations pesticides" (September 2019) <https://www.generationsfutures.fr/wp-content/uploads/2019/09/rapport_carences_evaluation_pesticides_100919.pdf>.

(2) ClientEarth, "REACh registration and endocrine disrupting chemicals" (1 July 2013) <https://www. documents.clientearth.org/library/download-info/reach-registration-and-endocrine-disrupting-chemicals/> .

(3) European Environmental Bureau (EEB) and ClientEarth, "Identifying bottlenecks in REACh implementation" (2012) < https://www.documents.clientearth.org/library/download-info/identifying-the-bottlenecks-in-reachimplementation/>. 
only increasing). The TGs have a handful of other huge insensitivities, including that they do not allow for the latency of chronic diseases (animals are killed at the human equivalent of approximately sixty years), they use archaic endpoints (light microscopy only) that miss untold numbers of effects, and they abuse "historic negative control" animals (replacing the experiment's actual control animals) to hide what inconvenient findings do appear. See Tweedale (2017; peer reviews) ${ }^{5}$ for more on these TG insensitivities.

The inability of any RA stakeholder to understand the necessity of beginning by evaluating AAI boggles the mind - for almost ten years, proposing this tactic has been rejected hundreds of times, and with point-blank refusals to begin any discussion. To the non-governmental organisations and scientists of Robinson et al, and to all other concerned parties, including regulators, scientists and policymakers: to achieve your desiderata, you must abandon your holistic/multifactorial problem construct, to think linearly. Backs turned are minds closed. This deserves discussion.

5 AC Tweedale, "The inadequacies of pre-market chemical risk assessment's toxicity studies - the implications" (2017) 37 Journal of Applied Toxicology 92-104. 